\title{
Parámetros reproductivos de vicuñas (Vicugna vicugna) en la Región Cusco, Perú
}

\author{
Reproductive parameters of vicuñas (Vicugna vicugna) in the Cusco region, Peru \\ Joel Iván Pacheco ${ }^{1,3}$, Víctor Velez ${ }^{1}$, Danilo Pezo ${ }^{1}$, José Angulo-Tisoc ${ }^{1}$, \\ Henry Castelo ${ }^{2}$
}

\section{Resumen}

El objetivo del presente estudio fue determinar algunos parámetros reproductivos (tasa de gestación, número de crías, distribución etaria y edad reproductiva) de vicuñas hembras en dos sistemas de manejo: silvestría y cautiverio, en la Región Cusco, Perú. Las capturas de los animales («Chaccus») se realizaron entre mayo y agosto de 2017. La gestación se determinó mediante ecografía transrectal en 143 hembras, la presencia de crías mediante la observación de ubre funcional en las hembras y la distribución etaria y edad reproductiva mediante las fichas de captura y observación de la dentición. El porcentaje de gestación fue de 74.6 y $79.4 \%$ para silvestría y cautiverio respectivamente. En la población juvenil se encontraba gestante el 29.9 y $4.4 \%$ en silvestría y cautiverio, respectivamente. El número de crías en silvestría fue de $36 \%$ y en cautiverio de $53 \%$. La proporción de crías fue similar en ambos sistemas de manejo, de juveniles fue de $20.1 \%$ en silvestría y $3.3 \%$ en cautiverio y de 84.9 y $66.3 \%$ en adultos en cautiverio yen silvestría, respectivamente.

Palabras clave: gestación; parámetros reproductivos; sistema de manejo; vicuña

\section{Abstract}

The aim of this study was to determine some reproductive parameters (gestation rate, number of young, age distribution and reproductive age) of female vicuñas in two management systems: wild and captivity, in the Cusco Region, Peru. The capture of the animals («Chaccus») were carried out between May and August 2017. The pregnancy

\footnotetext{
${ }^{1}$ Estación IVITA Marangani, Facultad de Medicina Veterinaria, Universidad Nacional Mayor de San Marcos, Cusco, Perú

2 Proyecto de Manejo y Uso Sustentable de la Vicuña del Gobierno Regional Cusco, Perú

${ }^{3}$ E-mail:jpachecoc@unmsm.edu.pe
} 
was determined by transrectal ultrasound in 143 females, the presence of offspring by observing functional udder in the females, the age distribution and reproductive age by means of the records of capture and observation of the dentition. The percentage of gestation was of 74.6 and $79.4 \%$ for the wild and captivity systems, respectively. In the juvenile population were pregnant 29.9 and $4.4 \%$ in the wild and in captivity, respectively. The number of young animals in the wild was $36 \%$ and in captivity $53 \%$. The proportion of young vicuñas was similar in both management systems, of juveniles was $20.1 \%$ in captivity and $3.3 \%$ in the wild and 84.9 and $66.3 \%$ in adults in captivity and in the wild, respectively.

Key words: gestation; management system; reproductive parameters; vicuña

\section{INTRODUCCIÓN}

La vicuña (Vicugna vicugna) es una especie polígama, forma grupos familiares con un macho dominante y varias hembras y crías, alcanza la madurez sexual al año y el primer parto lo pueden tener a los dos años; presentando actividad reproductiva durante la época lluviosa, de enero a marzo (Brack, 1980; Pérez, 1994). Se indica que la vicuña es la especie silvestre antecesora de la alpaca (Vicugna pacos) (Gentry et al., 2004), por cuanto ambas especies presentan muchas similitudes cercanas en cuanto a su fisiología reproductiva (Vaughan, 2011)

Es una especie protegida por el Estado mediante Ley N. ${ }^{\circ} 29763$, donde se indica que el Estado cumple un rol promotor, normativo y supervisor de los procesos de conservación y manejo, así como sobre el aprovechamiento de la fibra de los camélidos silvestres (SERFOR, 2015).

La población de vicuñas, según el último censo nacional de 2012 indica la existencia de 208899 animales, de los cuales el $69.9 \%$ se encuentra en silvestría y el $30.1 \%$ en semicautiverio (cercos permanentes), correspondiendo a la Región Cusco 17833 vicuñas censadas (DGFFS, 2014). Con el fin de incrementar el número de animales que puedan producir fibra en beneficio de las comunidades conservacionistas se implementaron los Módulos de Uso Sustentable (MUS), ahora denominados cercos permanentes, para lo cual existen los lineamientos técnicos de extracción y traslado de camélidos silvestres con fines de repoblamiento (DGFFS, 2012).

La actividad ovárica fue descrita en vicuñas argentinas ( $V$. vicugna vicugna), reportándose ondas foliculares de 7.2 días de duración y alternancia ovárica, similar a lo descrito en camélidos domésticos, pero con una mayor frecuencia del folículo dominante en el ovario derecho $(60 \%$ en el ovario derecho); además, la fase de crecimiento y la fase estática duraron 3 y 1.4 días en promedio, respectivamente y la regresión en 2.9 días (Miragaya et al., 2004). Mediante observaciones de vicuñas en cautiverio, se pudo determinar que el $74 \%$ de hembras tienen una cría a los 2 años y el $26 \%$ a los tres años; además, el $76 \%$ de hembras tuvo 6 crías en 10 años, y el $16 \%$ tuvo solo 5 crías en ese mismo periodo (Zúñiga, 2003a,b).

En condiciones de confinamiento se requiere que la vicuña tenga más de $40 \mathrm{~kg}$ de peso vivo para asegurar una gestación a término (Urquieta et al., 2012). El porcentaje de natalidad de vicuñas en silvestría en vicuñas de Argentina se reporta entre 51 y $68 \%$ (Arzamendia y Vilá, 2006) y de $62 \%$ en el norte de Chile (Gautier, 2004); asimismo, se describe $11.9 \%$ de pérdidas fetales en vicuñas mantenidas en cautiverio (Aller et al., 2003). Por otro lado, la fertilidad anual en guanacos en cautiverio fue entre 60 y $86.4 \%$ 
Cuadro 1. Porcentajes de gestación en vicuñas silvestres y en cautiverio (mayo -agosto, 2017)

\begin{tabular}{cccc}
\hline & Juvenil & Adulta & Total \\
\hline $\begin{array}{c}\text { Silvestría } \\
(\mathrm{n}=75)\end{array}$ & 29.3 & 44.3 & 74.6 \\
$\begin{array}{c}\text { Cautiverio } \\
(\mathrm{n}=68)\end{array}$ & 4.4 & 75 & 79.4 \\
\hline
\end{tabular}

en cuatro años de observación (Riveros et al., 2003).

El presente trabajo tuvo como objetivo determinar el porcentaje de gestación, número de crías, distribución etaria y edad reproductiva de vicuñas hembras de acuerdo con el tipo de manejo (silvestría y cautiverio), en la Región Cusco, Perú.

\section{Materiales y Métodos}

El estudio se realizó entre mayo y agosto de 2017 en las capturas («Chaccus») de vicuñas mantenidas en silvestría (cinco chaccus) y cautiverio (tres chaccus) en la Región Cusco. Se evaluó el total de hembras en cada captura en el caso de silvestría, mientras que en cautiverio (cerco permanente) se evaluaron 23 hembras por captura, seleccionadas al azar del corral de captura.

La gestación ( 75 hembras en silvestría y 68 hembras en cautiverio) se diagnosticó mediante ecografía transrectal utilizando un ecógrafo veterinario de $5 \mathrm{MHz}$ (Aloka ${ }^{\circledR} \mathrm{SSD}$ 280). El número de crías se determinó por la presencia de ubre funcional al momento de la evaluación y la edad se determinó a través de la dentadura (cría: dientes de leche; juvenil: dos dientes; adulto: 4 dientes en adelante). Así mismo, se determinaron características morfológicas como tamaño corporal y tipo de vellón entre grupos etarios. Los datos solo fueron analizados mediante estadística descriptiva.

El estudio fue amparado mediante una autorización de investigación a través de la Resolución de la Dirección General N. ${ }^{\circ} 180$ 2016-SERFOR/DGGSPFFS, en coordinación con el Proyecto de Manejo y Uso Sustentable de la Vicuña del Gobierno Regional Cusco y la Dirección Regional de Agricultura Cusco, así como con el personal de la ATFFSSERFOR Cusco y las comunidades conservacionistas de vicuñas.

\section{Resultados}

El porcentaje de gestación fue de 74.6 y $79.4 \%$ para vicuñas en silvestría y cautiverio, respectivamente (Cuadro 1); así mismo, se observó durante los chaccus que el 36 y $53 \%$ de madres adultas tenían crías lactantes para silvestría y cautiverio, respectivamente (Cuadro 2).

La distribución etaria fue similar en ambos sistemas de manejo para crías, mientras que la población adulta fue mayor bajo condiciones de crianza en cautiverio (Cuadro 3). En este cuadro se hace referencia al total de los animales capturados, incluyendo machos y hembras.

\section{Discusión}

El porcentaje de gestación en vicuñas mantenidas tanto en silvestría como en cautiverio fue de $77 \%$, valor superior a lo descrito para vicuñas en Argentina y Chile (Aller et al., 2003; Gautier, 2004; Arzamendia y Vilá, 2006). Este porcentaje se encuentra dentro del rango descrito para guanacos silvestres (Riveros et al., 2003). Los grupos familiares se encuentran todo el año con presencia de un macho dominante, el cual realiza la cópula durante la estación de monta, por ser una especie de comportamiento reproductivo estacional (Brack, 1980; Pérez, 1994). La 
Cuadro 2. Porcentaje de gestación y presencia de crías en vicuñas criadas en silvestría ( $n=7 \xi$ y en cautiverio $(n=68)$, de acuerdo con el grupo etario (mayo-agosto, 2017)

\begin{tabular}{cccccc}
\hline & \multicolumn{2}{c}{ Vacía } & \multicolumn{2}{c}{ Preñada } \\
\cline { 3 - 6 } & & Sin cría & Con cría & Sin cría & Con cría \\
\hline \multirow{2}{*}{ Silvestría } & Juvenil $(\mathrm{n}=30)$ & 10.6 & 0 & 29.4 & 0 \\
& Adulta $(\mathrm{n}=45)$ & 2.6 & 12 & 21.4 & 24 \\
Cautiverio & Juvenil $(\mathrm{n}=7)$ & 5.8 & 0 & 4.5 & 0 \\
& Adulta $(\mathrm{n}=61)$ & 5.8 & 8.8 & 30.9 & 44.2 \\
\hline
\end{tabular}

Cuadro 3. Distribución etariay por sexode vicuñas según el sistema de manejo(mayo-agosto, 2017)

\begin{tabular}{lccccc}
\hline \multirow{2}{*}{ Manejo } & \multicolumn{3}{c}{ Grupo etario, $\mathrm{n}(\%)$} & \multicolumn{2}{c}{ Sexo, $\mathrm{n}(\%)$} \\
\cline { 2 - 6 } & Cría & Juvenil & Adulto & Macho & Hembra \\
\hline Silvestría & $78(13.6)$ & $115(20.1)$ & $380(66.3)$ & $307(53.6)$ & $266(46.4)$ \\
Cautiverio & $126(11.8)$ & $35(3.3)$ & $904(84.9)$ & $541(50.8)$ & $524(49.2)$ \\
\hline
\end{tabular}

presencia continua del macho es similar al sistema de empadre tradicional de alpacas, donde los machos se mantienen en el rebaño con las hembras durante la época reproductiva, obteniéndose una fertilidad de 53-63\% (Guispe, 1996). Esto podría sugerir que las vicuñas presentarían una mayor eficiencia reproductiva estacional (Franklin, 1974, 2012).

El porcentaje de gestaciones en vicuñas adultas fue mayor en cautiverio (75.1\%) que en silvestría (45.4\%), mientras que Aller et al. (1998) reportaron $50 \%$ de fertilidad en vicuñas de la puna argentina. Una posible causa para este comportamiento podría ser la mayor cantidad de machos en cautiverio que, al no poder migrar normalmente, estarían más activos para buscar hembras y formar grupos familiares (Franklin, 1974), presentando una mayor actividad sexual y mayores posibilidades para fecundar a las hem- bras (Urquieta et al., 1994). En el caso de las hembras juveniles, el porcentaje de gestantes fue mayor en silvestría (29.4\%) respecto al cautiverio $(4.4 \%)$, donde probablemente existan otros factores que estén afectando este comportamiento sexual, tales como el factor nutricional y el estrés, puesto que en el sistema de cercos permanentes existe sobrepastoreo y baja calidad alimenticia en general (Siguas et al., 2011; Miranda et al., 2012), siendo la vicuña una especie que es selectiva en su dieta (Borgnia et al., 2010).

La tasa de gestación en hembras adultas con y $\sin$ cría fue de 24.0 y $12.0 \%$, respectivamente, en silvestría y de 44.2 y $8.8 \%$, respectivamente, en cautiverio, a diferencia del estudio de Aller et al. (1998) donde no encuentran diferencias entre animales gestantes con y sin cría al pie. En el presente estudio se repite el mismo patrón, tanto en 
silvestría y cautiverio, en donde el mayor porcentaje de crías perteneció a hembras adultas preñadas. Por otro lado, no se registraron hembras juveniles con cría.

Las tasas de gestación de 29.4 y $4.5 \%$ en vicuñas juveniles para silvestría y cautiverio respectivamente, estaría indicando que el inicio de su actividad reproductiva posiblemente se produciría antes de lo que indican otros autores (Brack, 1980; Pérez, 1994), y más temprano a lo reportado en alpacas (1 a 2 años), en donde se determinó un fuerte efecto del peso vivo en la actividad reproductiva (Vaughan, 2011; Pacheco et al., 2017).

Las crías representaron el 13.6 y $11.8 \%$ de la población total en silvestría y cautiverio, respectivamente, similar a reportes anteriores (Franklin, 1974), aunque superior al 3$8 \%$ reportado en una zona reservada en Argentina (Borgnia et al., 2006). Así mismo, la distribución etaria de las poblaciones de vicuñas juveniles difiere de acuerdo al tipo de manejo, encontrándose muy pocos juveniles en cautiverio respecto a silvestría, a pesar de que la presencia de crías fue similar, lo cual indicaría la existencia de otros factores que influyan en esta disminución, posiblemente enfermedades o depredadores (Arzamendia y Vilá, 2006), así como muertes causadas por la agresividad de los machos durante la época reproductiva (Franklin, 1974) y el estrés continuo ante la falta de espacio para poder migrar dentro del cerco permanente (Siguas et al., 2011).

\section{Agradecimientos}

Los autores agradecen al MVZ Leónidas Coila y al Sr Flavio Chipana de la Agencia Agraria Canchis-Cusco por las facilidades brindadas durante la obtención de muestras y datos, así como a Jhon Achicahuala y Ronal Rojas, biólogos del SERFOR Cusco.

\section{Literatura Citada}

1. Aller JF, Rebuffi GE, Kancino AK, Alberio RH. 1998. Diagnóstico de gestación por medio de ultrasonografía en vicuñas (Vicugna vicugna) en la puna argentina. Vet Arg 15: 504-509.

2. Aller JF, Rebuffi GE, Cancino AK, Alberio RH. 2003. Fetal mortality diagnosis by ultrasound in the vicuña (Vicugna vicugna). Reprod Fert Develop 15: 125-128.

3. Arzamendia Y, Vilá B. 2006. Estado y tendencia poblacional de vicuñas silvestres bajo manejo. En: IV Congreso Mundial sobre Camélidos. Catamarca, Argentina.

4. Borgnia M, Vilá B, Cassini M. 2006. Estimación poblacional local y estructura social de vicuñas en la reserva laguna blanca (Catamarca, Argentina). En: IV Congreso Mundial sobre Camélidos. Catamarca, Argentina.

5. Borgnia M, Vilá B, Cassini MH. 2010. Foraging ecology of vicuña, Vicugna vicugna, in dry puna of Argentina. Small Ruminant Res 88: 44-53. doi: 10.1016/ j.smallrumres.2009.11.009

6. Brack A. 1980. Conservación de la vicuña en el Perú - Proyecto especial de utilización racional de la vicuña. Información básica. Lima, Perú: Ministerio de Agricultura y Alimentación. 33 p.

7. [DGFFS] Dirección General Forestal y de Fauna Silvestre. 2012. Lineamientos técnicos de extracción y traslado de camélidos sudamericanos silvestres con fines de repoblamiento. Ministerio de Agricultura. Lima, Perú. 21 p.

8. [DGFFS] Dirección General Forestal y de Fauna Silvestre. 2014. Censo poblacional de vicuñas 2012. Ministerio de Agricultura y Riego. Lima, Perú. [Internet]. Disponible en: http:// www.agrolalibertad.gob.pe/sites/default/ files/Censo $\% 20$ Poblacional $\% 20$ de\%20Vicunas\%202012.pdf 
9. Franklin W. 1974. The social behaviour of the vicuña. In: The behaviour of ungulates and its relation to management. Switzerland: I.U.C.N. p 477-487.

10. Franklin W. 2012. Vicuña and guanaco behavioural ecology: a necessary understanding for their conservation \& management. In: VI Congreso Mundial sobre Camélidos Sudamericanos. Arica, Chile.

11. Gautier C. 2004. Gestación en vicuñas: fetometría ecográfica y esteroides ováricos maternos. Tesis de Médico Veterinario. Chile: Univ. de Chile. $40 \mathrm{p}$.

12. Gentry A, Clutton-Brock J, Groves CP. 2004. The naming of wild animal species and their domestic derivatives. JArchaeol Sci 31: 645-651. doi: 10.1016/ j.jas.2003.10.006

13. Guispe TL. 1996. Sistemas de empadre en alpacas. Rev Argent Prod Anim 16: 357-361.

14. Miragaya MH, Aba MA, Capdevielle EF, Ferrer MS, Chaves MG Rutter B, Aguero A. 2004. Follicular activity and hormonal secretory profile in vicuna (Vicugna vicugna). Theriogenology 61: 663-671. doi: 10.1016/S0093-691X(03)-00238-3

15. Miranda E, Cortes A, Miranda E. 2012. Composición de la dieta de la vicuña (Vicugna vicugna) en el altiplano del norte de Chile. En: VI Congreso Mundial sobre Camélidos Sudamericanos. Arica, Chile.

16. Pacheco JI, Pezo D, Vélez V, Bravo W. 2017. Descripción ecográfica del inicio de la actividad ovárica en alpacas (Vicugna pacos). Rev Invest Altoandin 2:195-200. doi: 10.18271/ria.2017.278

17. Pérez W. 1994. La saga de la vicuña. Lima, Perú: CONCYTEC. 408 p.

18. Riveros J, Bonacic C, Bas F, Gonzales B. 2003. Parámetros reproductivos de guanacos (Lama guanicoe) en cautiverio. En: III Congreso Mundial sobre Camélidos. Potosí, Bolivia.
19. [SERFOR] Servicio Nacional Forestal y de Fauna Silvestre. 2015. Resolución de Dirección Ejecutiva N. ${ }^{\circ} 060$ SERFOR-DE 2015. Lima Perú. [Internet]. Disponible en https://www.serfor.gob.pe/wp-content/uploads/2016/04/ RE S O L U C I \% C $3 \% 93 \mathrm{~N}$ - D E DIRECCI\%C3\%93N-EJECUTIVAN\%C2\%BA-060-2016-SERFOR-DEAprobar-los-lineamientos-para-el-otorgamiento-de-la-autorizacion-con-finesde-investigacion-cientifica-de-flora-yfauna-silvestre.pdf

20. Siguas O, Olazábal J, Páucar R, Bonacic C. 2011. Respuesta de estrés de captura en vicuñas silvestres (Vicugna vicugna Thomas, 1917) en condiciones de puna peruana. En: VII Congreso de la Asociación Latinoamericana de Especialistas en Pequeños Rumiantes y Camélidos Sudamericanos - ALEPRyCS. Huancavelica, Perú.

21. Urquieta B, Cepeda $R$, Cáceres JE, Raggi LA, Rojas JR. 1994. Seasonal variation in some reproductive parameters of male vicuña in the High Andes of northern Chile. J Arid Environ 26: 79-87.

22. Urquieta B, Varas MH, Parraguez $V H$, Raggi LA. 2012. Gestación y peso corporal en vicuñas en confinamiento en el altiplano del extremo norte de Chile. En: VICongreso Mundial sobre Camélidos Sudamericanos. Arica, Chile.

23. Vaughan J. 2011. Ovarian function in south American camelids (alpacas, llamas, vicuñas, guanacos). Anim Reprod Sci 124: 237-243. doi: 10.1016/j.anireprosci.2010.08.031

24. Zúñiga M. 2003a. Estudios complementarios de la biología de la vicuña. En: III Congreso Mundial sobre Camélidos. Potosí, Bolivia.

25. Zúñiga M. 2003b. Ciclo reproductivo de la vicuña. En: III Congreso Mundial sobre Camélidos. Potosí, Bolivia. 